\title{
NuSTAR reveals that the heavily obscured nucleus of NGC 2785 was the contaminant of IRAS 09104+4109 in the BeppoSAX/PDS hard X-rays
}

\author{
C. Vignali ${ }^{1,2}$, P. Severgnini ${ }^{3}$, E. Piconcelli ${ }^{4}$, G. Lanzuisi ${ }^{1,2}$, R. Gilli ${ }^{2}$, M. Mignoli ${ }^{2}$, A. Comastri ${ }^{2}$, L. Ballo $^{5}$, \\ K. Iwasawa ${ }^{6,7}$, and V. La Parola ${ }^{8}$ \\ 1 Dipartimento di Fisica e Astronomia, Alma Mater Studiorum, Università degli Studi di Bologna, Via Gobetti 93/2, \\ 40129 Bologna, Italy \\ e-mail: cristian.vignali@unibo.it \\ 2 INAF - Osservatorio di Astrofisica e Scienza dello Spazio di Bologna, Via Gobetti 93/3, 40129 Bologna, Italy \\ 3 INAF - Osservatorio Astronomico di Brera, Via Brera 28, 20121 Milano, Italy \\ 4 INAF - Osservatorio Astronomico di Roma, Via Frascati 33, 00040 Monteporzio Catone, Roma, Italy \\ 5 XMM-Newton Science Operations Centre, ESAC/ESA, PO Box 78, 28691 Villanueva de la Canãda, Madrid, Spain \\ 6 Institut de Ciències del Cosmos (ICCUB), Universitat de Barcelona (IEEC-UB), Martí Franquès, 1, 08028 Barcelona, Spain \\ 7 ICREA, Pg. Lluís Companys 23, 08010 Barcelona, Spain \\ 8 INAF - Istituto di Astrofisica Spaziale e Fisica Cosmica di Palermo, Via Ugo La Malfa 153, 90146 Palermo, Italy
}

Received 9 July 2018 / Accepted 6 August 2018

\begin{abstract}
Context. The search for heavily obscured active galactic nuclei has been revitalized in the last five years by NuSTAR, which has provided a good census and spectral characterization of a population of such objects, mostly at low redshift, thanks to its enhanced sensitivity above $10 \mathrm{keV}$ compared to previous X-ray facilities, and its hard X-ray imaging capabilities.

Aims. We aim at demonstrating how NGC 2785, a local $(z=0.009)$ star-forming galaxy, is responsible, in virtue of its heavily obscured active nucleus, for significant contamination in the non-imaging BeppoSAX/PDS data of the relatively nearby $\left(\approx 17^{\prime}\right)$ quasar IRAS $09104+4109(z=0.44)$, which was originally mis-classified as Compton thick.

Methods. We analyzed $\approx 71 \mathrm{ks} \mathrm{NuSTAR}$ data of NGC 2785 using the MYTorus model and provided a physical description of the X-ray properties of the source for the first time.

Results. We found that NGC 2785 hosts a heavily obscured $\left(N_{\mathrm{H}} \approx 3 \times 10^{24} \mathrm{~cm}^{-2}\right)$ nucleus. The intrinsic X-ray luminosity of the source, once corrected for the measured obscuration $\left(L_{2-10 \mathrm{keV}} \approx 10^{42} \mathrm{erg} \mathrm{s}^{-1}\right)$, is consistent within a factor of a few with predictions based on the source mid-infrared flux using widely adopted correlations from the literature.

Conclusions. Based on NuSTAR data and previous indications from the Neil Gehrels Swift Observatory (BAT instrument), we confirm that NGC 2785, because of its hard X-ray emission and spectral shape, was responsible for at least one third of the 20-100 keV emission observed using the PDS instrument onboard BeppoSAX, originally completely associated with IRAS 09104+4109. Such emission led to the erroneous classification of this source as a Compton-thick quasar, while it is now recognized as Compton thin.
\end{abstract}

Key words. galaxies: active - X-rays: galaxies - X-rays: individuals: NGC 2785

\section{Introduction}

Currently, there is a wide consensus on the fact that a fraction of $\sim 50 \%$ of Seyfert 2 galaxies in the local Universe are obscured in the X-ray band by column densities of the order of, or larger than, the inverse of the Thomson cross-section $\left(N_{\mathrm{H}} \geq \sigma_{\mathrm{T}}^{-1} \simeq 1.5 \times 10^{24} \mathrm{~cm}^{-2}\right)$; these sources are called Compton thick (CT; e.g., Matt 2000; Guainazzi et al. 2005). If the optical depth $\left(\tau=N_{\mathrm{H}} \sigma_{\mathrm{T}}\right)$ for Compton scattering does not exceed values of the order of a few, X-ray photons with energies higher than $10-15 \mathrm{keV}$ are able to penetrate the obscuring material and reach the observer. For higher values of $\tau$, the whole X-ray spectrum is depressed by Compton down-scattering, and the X-ray photons are effectively trapped by the obscuring material irrespective of their energy. The former class of sources (mildly CT) can be efficiently detected by X-ray instruments sensitive above $10 \mathrm{keV}$, while the nature of the latter (heavily CT) can be inferred through indirect arguments, such as the presence of a strong iron $\mathrm{K} \alpha$ line over a flat reflected continuum, or via observations/selections at other wavelengths (e.g., in the mid infrared; see e.g., Vignali 2014; and references therein).

The search for and the study of the physical properties of CT active galactic nuclei (AGNs) is relevant to understand the evolution of accreting super-massive black holes (SMBHs). In particular, mildly CT AGNs are the most promising candidates to explain the residual (i.e., not yet resolved) spectrum of the cosmic X-ray background around its $30 \mathrm{keV}$ peak (e.g., Gilli et al. 2007; Treister et al. 2009; Ballantyne et al. 2011; Akylas et al. 2012). The existence of CT objects indicates that we may be missing a non-negligible fraction of the accretion power in the Universe and of the baryonic matter locked in SMBHs (Marconi et al. 2004; Comastri et al. 2015). Because of the highly depressed emission (due to photoelectric absorption) at energies below $\approx 10 \mathrm{keV}$, the detection of CT AGN is often still a challenge. 
The hard X-ray band up to $\approx 100-150 \mathrm{keV}$ is potentially appropriate to provide an almost unbiased census of obscured AGNs, since both Compton-thin and CT AGN (with column densities up to $\approx 10^{25} \mathrm{~cm}^{-2}$ ) can be detected by hard X-ray instruments; however, the sensitivity of the Neil Gehrels Swift Observatory/BAT and Integral/IBIS surveys $\left(\approx 10^{-11} \mathrm{erg} \mathrm{cm}^{-2} \mathrm{~s}^{-1}\right.$; e.g., Tueller et al. 2008; Beckmann et al. 2009; Burlon et al. 2011; Malizia et al. 2012) limits this kind of investigation to the local Universe and results in a few per cent of the XRB being resolved into discrete sources at its peak around $20-30 \mathrm{keV}$ (e.g., Ajello et al. 2012; Vasudevan et al. 2013). These studies often require follow-up observations with more sensitive instruments to define the source properties in a more exhaustive and comprehensive way (e.g., Severgnini et al. 2011).

Over the last five years NuSTAR, thanks to its much higher sensitivity (a factor of $\approx 100$ above $10 \mathrm{keV}$ ) compared to the hard X-ray instruments cited above, coupled with focusing optics in the hard band, has extended the search for heavily obscured AGNs up to $z \sim 3$ for a limited number of sources (Del Moro et al. 2017; Lansbury et al. 2017a) and is providing a more complete census than before of the obscured AGN population at low redshift (e.g., Baloković et al. 2014; Gandhi et al. 2014; Lansbury et al. 2015, 2017b; Ricci et al. 2015; Masini et al. 2016; Boorman et al. 2016; Marchesi et al. 2018; Zappacosta et al. 2018).

Here we present the X-ray properties of the heavily obscured nucleus of NGC 2785, a disky galaxy at $z=0.009$, derived from a $71 \mathrm{ks} N U S T A R$ observation. Our interest in this source takes its origin primarily from Vignali et al. (2011, V11). In that work, the analysis of the 54-month Swift/BAT map in the $15-30 \mathrm{keV}$ band suggested that the hard X-ray emission, detected by the non-imaging PDS instrument onboard BeppoSAX during the observation of the luminous quasar IRAS 09104+4109 (hereafter I09104, at $z=0.442$ ) and originally associated with I09104 (Franceschini et al. 2000), was most likely spatially coincident with NGC 2785. The distance between I09104 and NGC 2785 positions is about 17' (see Fig. 2 of V11). The intensity of the hard X-ray emission tentatively detected $(\approx 3 \sigma)$ by BeppoSAX/PDS and associated with I09104, coupled with the presence of an iron $\mathrm{K} \alpha$ emission line with large equivalent width $(\mathrm{EW} \approx 1-2 \mathrm{keV})$, was originally used to classify I09104 as a CT quasar (Franceschini et al. 2000), making de facto I09104 the prototype for luminous, high-redshift CT quasars for many years.

Doubts on the classification of I09104 as a CT AGN were cast at first by Piconcelli et al. (2007; P07 hereafter) using $X M M$-Newton data, where the large iron EW measured by BeppoSAX/MECS was ascribed to the presence of blended iron emission lines (a neutral iron line from the AGN and highly ionized iron transitions from the hot gas of the surrounding cluster of which I09104 is the cD galaxy). Subsequently, Chiang et al. (2013), using Suzaku data, confirmed the Compton-thin nature of the absorber in I09104 (see also Farrah et al. 2016, who collected the available data for I09104, including NuSTAR). Unfortunately, NGC 2785 does not fall within the field of view of any of the available X-ray datasets for I09104 (Chandra; see also Iwasawa et al. 2001; XMM-Newton, Suzaku, and NuSTAR), thus preventing a secure association of this source as the "contaminant" in BeppoSAX/PDS data of I09104.

From the optical perspective, the analysis of the Sloan Digital Sky Survey (SDSS) spectrum ${ }^{1}$ of NGC 2785 shows a very

\footnotetext{
1 http://skyserver.sdss.org/dr14/en/tools/chart/navi. aspx
}

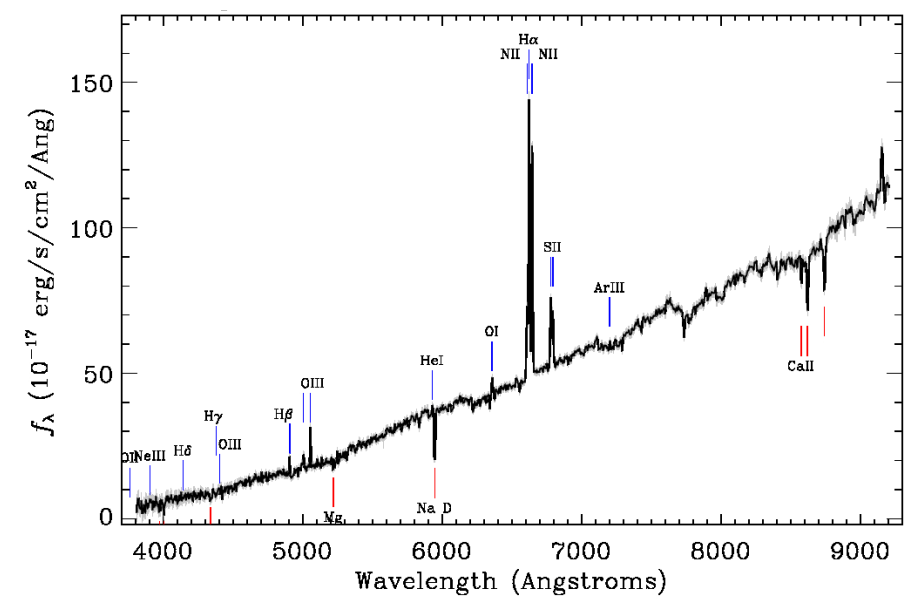

Fig. 1. SDSS spectrum of NGC 2785. The source is characterized by a very red continuum and a large $\mathrm{H} \alpha / \mathrm{H} \beta$ line ratio (even after correction for the host galaxy contribution), suggestive of a heavily extinguished object (see text and V11 for details). The main emission/absorption lines (as reported in the SDSS webpage) are labeled.

red continuum and narrow optical emission lines (see Fig. 1 and V11). While the presence of the AGN is inferred from the $\mathrm{H} \alpha /[\mathrm{NII}]$ and $\mathrm{H} \beta /[\mathrm{OIII}]$ line ratios (e.g., Veilleux et al. 1995), the red continuum is indicative of the dominant contribution of the dust-rich host galaxy at optical wavelengths, with $E(B-V) \approx$ 1.5-1.9 assuming a Galactic extinction law (Seaton 1979). This represents the extinction within the host galaxy, not related to the nuclear regions. At face value, NGC 2785 can be considered a low-redshift analog of dust-obscured galaxies at $z \approx 2-3$ (e.g., Stern et al. 2014; Piconcelli et al. 2015), where a heavily obscured nucleus is often hidden in a star-forming galaxy.

The present NUSTAR observation is meant to shed definitive light on the picture described above. In particular, to overcome the limited significance $(\approx 3.5 \sigma$ level $)$ of the excess emission in the Swift/BAT map presented by V11, hard X-ray imaging and sensitive data are highly required.

In this paper we present $N U S T A R$ data reduction, analysis, and interpretation in Sect. 2, while in Sect. 3 further multiwavelength support to the nature of NGC 2785 as a Comptonthick AGN is reported, providing further indication that it was a contaminant of the BeppoSAX/PDS flux observed in I09104. Conclusions are presented in Sect. 4.

The adopted cosmology consists of $H_{0}=70 \mathrm{~km} \mathrm{~s}^{-1} \mathrm{Mpc}^{-1}$, $\Omega_{\Lambda}=0.73$ and $\Omega_{\mathrm{M}}=0.27$. Errors are reported at the $90 \%$ confidence level for one parameter of interest (Avni 1976), unless stated otherwise.

\section{NuSTAR data}

\subsection{Data reduction and spectral extraction}

NGC $2785 \quad(\mathrm{RA}=09: 15: 15.4, \quad$ Dec $=40: 55: 03 ; \quad J 2000)$ was observed by NuSTAR (Harrison et al. 2013) for $72 \mathrm{ks}$ on May 5,2016 . The raw data were calibrated and filtered using the NuSTAR Data Analysis Software package (NuSTARDAS) v. 1.6.0 (included in HEASOFT v. 6.19), the calibration files in the NuSTAR CALDB (version 20160315), and standard settings for the NUPIPELINE task. Inspection of the FPMA and FPMB light curves in the 3-20 keV energy range shows the presence of a short-lived $(\approx 1 \mathrm{ks})$ background flare due to solar activity, which was removed using the nustar_filter_lightcurve IDL 


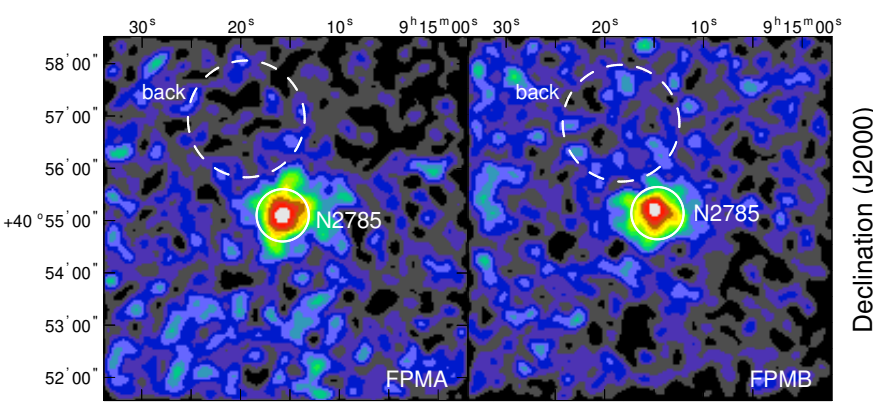

Right Ascension (J2000)

Fig. 2. NUSTAR images in the $4-30 \mathrm{keV}$ band for the two modules (FPMA on the left panel, FPMB on the right panel). The chosen source and background extraction regions, as reported in the text, are indicated by the solid and dashed circles, respectively.

script. The resulting cleaned exposure time is $71 \mathrm{ks}$ for both modules.

Source and background spectra (plus the corresponding response matrices) were extracted using the NUPRODUCTs task. Since NGC 2785 did not appear particularly bright from visual inspection of the NUSTAR images, we decided to favor the signalto-noise ratio $(\mathrm{S} / \mathrm{N})$ in the resulting spectra by choosing a circular source extraction region (in both modules) of radius $\mathrm{R}=30^{\prime \prime}$, while a larger circular $\left(\mathrm{R}=67^{\prime \prime}\right)$ region, free of field sources, was selected for the background (see Fig. 2). The source net (i.e., background subtracted) counts in the $\approx 4-30 \mathrm{keV}$ energy range are $\approx 280$ and $\approx 270$ in FPMA and FPMB, respectively. The two spectra were rebinned to have at least 20 total (i.e., source plus background) counts per bin in order to apply the $\chi^{2}$ statistics in the spectral analysis phase, performed using XSPEC (Arnaud 1996). The background is not dominant in either of the two spectra, with the net source contribution being $75-80 \%$ of the total counts over the entire NUSTAR energy band used in this work. However, we also checked whether the X-ray spectral results may depend on the adopted binning method. To this goal, we binned the two spectra to a $\mathrm{S} / \mathrm{N}$ of three and obtained spectral results which are fully consistent with those reported in the following analysis (Sect. 2.2). The two datasets used in the simultaneous X-ray spectral analysis are largely consistent, as confirmed by the cross-calibration constant being close to unity.

\subsection{X-ray spectral results}

A quick visual inspection of the extracted NuSTAR spectra suggests that NGC 2785 is characterized by a flat X-ray continuum coupled with a strong iron emission line, which are indicative of heavily obscured emission. To provide a preliminary, though phenomenological, description of the spectra, we adopted a model comprising an absorbed power law for the intrinsic hard X-ray continuum, a Gaussian line for iron emission, and an additional power law to account for the emission emerging at $E<10 \mathrm{keV}$, likely related (at least in part) to scattering (few per cent of the nuclear emission), as often observed in obscured nuclei (e.g., Lanzuisi et al. 2015a). The fit is relatively good $\left(\chi^{2} /\right.$ d.o.f. $\left.=25.3 / 27\right)$; the presence of extreme absorption (within the CT regime) is indicated by the derived column density of $\approx 2 \times 10^{24} \mathrm{~cm}^{-2}$ and the apparently strong EW $(\approx 1 \mathrm{keV})$ of the iron line. These results call for a more physically motivated modeling of the data.

Dealing with a CT AGN candidate, to define the intrinsic continuum of NGC 2785 and place constraints on the level of

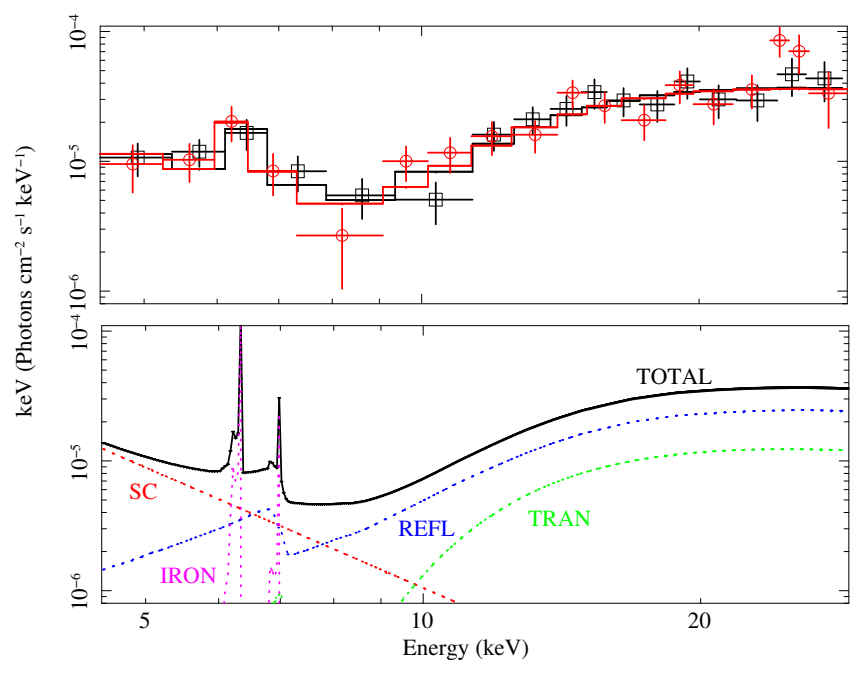

Fig. 3. Top panel: NuSTAR FPMA (black) and FPMB (red) unfolded spectra of NGC 2785, fitted using MYTorus and a soft component. Bottom panel: model components (TOTAL: best-fitting model; SC: scattering (power law) component; REFL/TRAN/IRON: reflected, transmitted and iron line components in the MYTorus modeling (see text and Murphy \& Yaqoob 2009).

obscuration we used the MYTonus model (Murphy \& Yaqoob 2009), which is based on Monte Carlo simulations; assuming a toroidal geometry for the reprocessor (uniform and cold), it self-consistently includes reflection and transmission (see e.g., Lanzuisi et al. 2015b for further details, and Appendix B of Lanzuisi et al. 2015a for a comparison with other models applied to heavily obscured AGN). An additional component, parameterized by a power law, was included in the spectral fitting to account for some emission at low energy (see below for details). We left the main parameters of the spectral fit with MYToRus free to vary, namely the photon index, the equatorial column density of the absorber, and the inclination angle between the observer's line of sight and the symmetry axis of the torus $\theta_{\mathrm{obs}}$; the reprocessor, by construction, has a fixed half-opening angle of $60^{\circ}$.

The best-fit spectral results $\left(\chi^{2} /\right.$ d.o.f. $\left.=26.5 / 28\right)$ shown in Fig. 3 indicate that the nucleus of NGC 2785 is well parameterized by a hard power law component with $\Gamma=1.85_{-0.17}^{+0.31}$, absorbed by a column density $N_{\mathrm{H}}=3.0_{-0.6}^{+1.6} \times 10^{24} \mathrm{~cm}^{-2}$, thus placing the source in the CT regime. We note that this column density value does not match - as expected - with the obscuration obtained converting the extinction measured in the SDSS spectrum (Sect. 1) using Bohlin et al. (1978) and the Galactic dust-to-gas ratio. This value is of the order of $10^{22} \mathrm{~cm}^{-2}$. In fact, while the SDSS spectrum provides a measurement of the extinction on galaxy scales, the X-ray-derived column density is dominated by the obscuration likely associated with the torus on much smaller (parsec) scales. The observer's line of sight intercepts the torus at an inclination angle of $79_{-10}^{+8}$ degrees, suggesting an almost equatorial view of the central engine.

The additional power law component (whose inclusion in the MYTorus model provides a $\Delta \chi^{2}=8.4$ for two additional degrees of freedom, corresponding to an F-test probability of $\approx 98 \%$ ) turns out to be a few per cent of the nuclear one and extremely steep $(\Gamma \approx 4)$ with loose constraints, largely because of the limited photon statistics and bandpass redward of the iron line energy. Reasonably, it may be a mixture of a scattered, AGN-related component, and thermal emission from the host galaxy, which we cannot model properly with the current data. 
The apparent steepness of such a component hints at the thermal emission hypothesis, although its extrapolated $0.5-2 \mathrm{keV}$ luminosity, assuming the Ranalli et al. (2003) relation, would imply a star-formation rate at least one order of magnitude higher than the one derived from the spectral energy distribution (SED) fitting described in Sect. 3.1. We also point out that a relatively good fit is still obtained if the photon index of the soft component is tied to that of the intrinsic component, with a difference in $\Delta \chi^{2}$ of $\approx 5$ with respect to the best-fitting solution described above. However, given the quality of the NuSTAR data and the limited residuals (some deviations are present at $\approx 25 \mathrm{keV}$ and are most likely instrumental), we decided not to complicate this model further. The observed $2-10 \mathrm{keV}(20-30 \mathrm{keV})$ flux is $\approx 2.6 \times 10^{-13} \mathrm{erg} \mathrm{cm}^{-2} \mathrm{~s}^{-1}\left(\approx 5.7 \times 10^{-13} \mathrm{erg} \mathrm{cm}^{-2} \mathrm{~s}^{-1}\right)$, which translates into an intrinsic (i.e., absorption-corrected) $2-10 \mathrm{keV}$ luminosity of $\approx 1.1 \times 10^{42} \mathrm{erg} \mathrm{s}^{-1}$; the ratio intrinsic/measured luminosity in the $20-30 \mathrm{keV}$ band is $\approx 25$.

\section{Discussion}

\subsection{The heavily obscured nucleus of NGC 2785}

In the following, we provide support to the heavily obscured nature of NGC 2785 revealed by NuSTAR using the available multi-wavelength information.

The analysis of its SDSS spectrum (see V11 and Sect. 1) allows us to estimate the strength of the de-reddened [O III] $5007 \AA$ emission line which, converted into a $2-10 \mathrm{keV}$ flux assuming relations by Mulchaey et al. (1994, or similar relations, with typical dispersions of $\approx 0.5 \mathrm{dex}$; see also Heckman et al. 2005; Panessa et al. 2006; LaMassa et al. 2011), provides a value of $(0.9-4.7) \times 10^{-11} \mathrm{erg} \mathrm{cm}^{-2} \mathrm{~s}^{-1}$ (the range is due to the uncertainties on the $E(B-V)$ ), that is, a factor $\approx 35-180$ higher than the one actually measured by NuSTAR. This is preliminary evidence, besides the X-ray spectral analysis, that the AGN in NGC 2785 is possibly strongly depressed below $10 \mathrm{keV}$.

Furthermore, the analysis of the source SED in terms of galaxy+AGN emission decomposition (along the lines described in e.g., V11, and references therein) provides a first-order estimate of the AGN emission in the mid-IR, which can be used as a proxy of the intrinsic (e.g., 2-10 keV) AGN strength. The SED fitting is based on a multicomponent analysis, including stars (having the bulk of the emission in the optical/near-IR); hot dust, mainly heated by UV/optical emission due to gas accreting onto the SMBH and whose emission peaks somewhere between a few and a few tens of microns; and cold dust, principally heated by star formation, peaking around $100 \mu \mathrm{m}$; see Fritz et al. (2006) and Feltre et al. (2012) for further insights into this modeling. Adopting mid-IR versus hard X-ray correlations for the AGN component (see, e.g., Lanzuisi et al. 2009; Gandhi et al. 2009; Severgnini et al. 2012; Asmus et al. 2015, and the recent parameterizations provided by Stern 2015 and Martocchia et al. 2017), it is possible to derive the expected, intrinsic $2-10 \mathrm{keV}$ luminosity. At $12 \mu \mathrm{m}$ (covered by WISE data) about one quarter of NGC 2785 flux is related to the AGN, while the remainder is due to the host galaxy, according to the SED decomposition described above. This translates into an intrinsic $2-$ $10 \mathrm{keV}$ luminosity of $\approx 2.5 \times 10^{42} \mathrm{erg} \mathrm{s}^{-1}$ assuming Eq. (2) of Gandhi et al. (2009) ${ }^{2}$. At face value (i.e., without considering

\footnotetext{
2 Gandhi et al. (2009) used high-resolution near-diffraction-limited observations in the mid-infrared to separate the host galaxy emission from the nuclear component in a sample of 42 local AGNs. Equation (2) refers to the subsample of 22 well-resolved sources.
}

the dispersion in the used correlation, $\approx 0.2 \mathrm{dex}$ ), the predicted luminosity is, within a factor of approximately two, consistent with the one derived from our best-fitting X-ray spectral solution (see Sect. 2.2), further supporting the results of our analysis of NuSTAR data.

As a further validation test, we used the AGN bolometric luminosity, obtained from the SED fitting described above, to estimate the bolometric correction in the $2-10 \mathrm{keV}$ band (defined as $k_{2-10 \mathrm{keV}}=L_{\mathrm{bol}} / L_{2-10 \mathrm{keV}}$, assuming the Lusso et al. 2012 relation for spectroscopically confirmed obscured AGNs in COSMOS). For NGC 2785, the estimated bolometric luminosity of $\approx 1.7 \times 10^{43} \mathrm{erg} \mathrm{s}^{-1}$ implies a bolometric correction of $\approx 15$. This means that the intrinsic $2-10 \mathrm{keV}$ luminosity would be $\approx 1.1 \times 10^{42} \mathrm{erg} \mathrm{s}^{-1}$, in agreement with the value derived from our X-ray spectral analysis once the obscuration is taken into account.

\subsection{The active role of NGC 2785 in contaminating BeppoSAX/PDS data}

As previously introduced, one of the goals of the current $N u S$ TAR observation of NGC 2785 was to understand whether, and at what level, its hard X-ray emission might be responsible for the tentative detection of I09104 in BeppoSAX/PDS nonimaging data $\left(20-100 \mathrm{keV}\right.$ flux of $1.0 \pm 0.3 \times 10^{-11} \mathrm{erg} \mathrm{cm}^{-2} \mathrm{~s}^{-1}$, Franceschini et al. 2000), making it the prototype for luminous, high-redshift CT quasars for many years. In a repeat analysis of the same data carried out by P07, I09104 was reported as a $2.5 \sigma$ detection in the $15-50 \mathrm{keV}$ band, with a flux of $2.55_{-1.56}^{+1.90} \times 10^{-12} \mathrm{erg} \mathrm{cm}^{-2} \mathrm{~s}^{-1}$ in the $20-30 \mathrm{keV}$ band. The same authors (and, in the following years, V11; Chiang et al. 2013; Farrah et al. 2016, using multiple X-ray datasets with I09104 as the target) have produced further evidence that I09104 was originally mis-classified as CT, and is in fact in the Compton-thin regime (with a column density of $\approx 4 \times 10^{23} \mathrm{~cm}^{-2}$ according to the Chandra analysis by V11). However, the "culprit" of the hard $\mathrm{X}$-ray contamination was still missing. The first piece of the puzzle towards a solution of this issue was placed by Swift/BAT, whose 54-month data in the $15-30 \mathrm{keV}$ band (V11) suggested that NGC 2785 could be the contaminant (lying at a distance of $\approx 17^{\prime}$ from I09104). However, the limited $\mathrm{S} / \mathrm{N}(\approx 3.5 \sigma$ in $\mathrm{V} 11$, being still below the $4.8 \sigma$ detection limit in the recently published 105-month analysis; Oh et al. 2018), combined with the poor positional accuracy in Swift/BAT data, prevented V11 from drawing firm conclusions about the association of NGC 2785 with the "excess flux" detected by BeppoSAX.

NuSTAR, because of its higher sensitivity and spatial resolution than previous hard X-ray missions, has been able to provide the "smoking gun": NGC 2785, in virtue of its heavily obscured nuclear emission emerging strongly in hard X-rays, is able to broadly justify the previously reported BeppoSAX flux, admittedly characterized by large uncertainties due to a marginal detection in the non-imaging PDS instrument. In fact, if we consider the combined $20-30 \mathrm{keV}$ flux produced by NGC 2785 (current NuSTAR data), and that relative to I09104 (Farrah et al. 2016, NuSTAR data), we obtain a value of $(5.7+$ $7.0) \times 10^{-13} \approx 1.3 \times 10^{-12} \mathrm{erg} \mathrm{cm}^{-2} \mathrm{~s}^{-1}$, which is consistent, within the errors, with the $\approx 2.6 \times 10^{-12} \mathrm{erg} \mathrm{cm}^{-2} \mathrm{~s}^{-1}$ reported by $\mathrm{P} 07$ from the repeat analysis of BeppoSAX/PDS data. If we compare the $20-100 \mathrm{keV}$ band flux of I09104 in the PDS (Franceschini et al. 2000) with the extrapolation of I09104 and NGC 2785 fluxes from NuSTAR data in the same energy range, $\approx 6 \times 10^{-12} \mathrm{erg} \mathrm{cm}^{-2} \mathrm{~s}^{-1}$, we can safely say that most of the highenergy flux detected by the PDS is accounted for. Some source 
variability and the presence of other lower-luminosity sources in the PDS field of view (see P07 and V11) can account for the low discrepancy between the two fluxes. As a side note, the angular response of the PDS collimators is $\sim 80 \%$ at the position of NGC 2785 in the BeppoSAX/PDS data of I09104 (see Fig. 2 of Frontera et al. 2007), meaning that the expected flux contribution of NGC 2785 reported above should not be significantly scaled down once the offset position of NGC 2785 with respect to I09104 is taken into account.

In the following we provide a rough estimate of the incidence of cases similar to the one described in this work "hidden" in old datasets taken by X-ray telescopes with non-focusing optics. In particular, we start focusing on BeppoSAX detections of CT AGN above $10 \mathrm{keV}$ using the compilation presented in the review by Comastri (2004). We complement this list with the one presented by Della Ceca et al. (2008). Although probably not complete, the final list is suitable to make an estimation of the fraction of sources that were originally classified as CT by BeppoSAX using $>10 \mathrm{keV}$ data but were probably contaminated by relatively nearby objects in the PDS (not necessarily CT as in our case). Five out of 25 AGNs of this list (20\%), including I09104, were not confirmed as CT using more recent datasets (in some cases comprising NuSTAR). On the one hand, $N U S T A R$ clearly has a relevant role in this kind of investigation at hard X-rays compared to non-imaging instruments because of its focusing optics and higher sensitivity; on the other hand, goodquality X-ray data below $10 \mathrm{keV}$ are often sufficient to shed some (maybe not conclusive) light on the nature of heavily obscured AGNs even in the absence of sensitive data at higher energies.

Recently, Marchesi et al. (2018) reported that Compton-thin AGNs detected by Swift/BAT (characterized by non-focusing optics) may have been mis-classified as CT (40\% in the original 70-month sample) mainly because of low-quality data, leading to a general over-estimation of the true intrinsic X-ray obscuration. This result, which was achieved using NuSTAR coupled with sensitive observations below $10 \mathrm{keV}$, suggests a slight revision of the fraction of CT AGNs in the Swift/BAT sample towards lower values (from 7.6\% in Ricci et al. 2015 compilation to 6\% - and, potentially, down to $4 \%$ - in the Marchesi et al. 2018 analysis). In this context, given the BeppoSAX results discussed above, we may argue that, overall, the impact of cases such as the one described in our work (contamination in the field of view of nonimaging instruments, causing the target to be detected as CT) on the cosmic X-ray background modeling is probably limited.

\section{Conclusions}

NGC 2785 represents a very good case of a buried AGN in a dusty galaxy, likely a low-redshift analog of the heavily absorbed AGN found in dust-obscured galaxies at $z \approx 2-3$. The $\approx 70 \mathrm{ks}$ $N u S T A R$ observation has allowed, for the first time, to obtain a medium-quality X-ray spectrum of this source, which was previously found to be highly extinguished on the basis of its SDSS spectrum alone. Based on an admittedly low $\mathrm{S} / \mathrm{N}$ detection in the 54-month Swift/BAT catalog (V11), the source was also supposed to be the contaminant of I09104 (an obscured quasar at $z=0.44$ ) flux in the non-imaging BeppoSAX/PDS hard X-ray data.

Applying the MYTorus model to the NUSTAR data has allowed us to place constraints on NGC 2785 nuclear emission, which is absorbed by a column density of $\approx 3 \times 10^{24} \mathrm{~cm}^{-2}$, and to derive the source's intrinsic luminosity as being $\approx 10^{42} \mathrm{erg} \mathrm{s}^{-1}$ in the $2-10 \mathrm{keV}$ band. This result places NGC 2785 among the "certified" CT AGNs. Further support to the presence of heavy obscuration (i.e., to the fact that intrinsically the source is much brighter than actually observed) comes from the de-reddened [O III] intensity, the AGN mid-IR flux, and the source bolometric luminosity (derived from SED fitting). The claim (V11) that this source could contaminate the BeppoSAX/PDS data of I09104 is fully corroborated by the current findings.

The results presented in this work clearly stress the need for sensitive imaging and spectroscopic observations at energies above $10 \mathrm{keV}$ to disclose heavily obscured nuclei. NUSTAR represents the most efficient way to provide a comprehensive picture of sources like NGC 2785 and useful information for the adequate interpretation of their optical/mid-infrared properties.

Acknowledgements. CV thanks the referee for her/his useful comments. The authors acknowledge financial support from the Italian Space Agency (ASI) under the contracts ASI-INAF I/037/12/0 and ASI-INAF n.2017-14-H.0. CV thanks M. Orlandini for a useful discussion about the response of the PDS instrument onboard BeppoSAX, and L. Zappacosta for indications about NuSTAR data analysis in the presence of flares.

\section{References}

Ajello, M., Alexander, D. M., Greiner, J., et al. 2012, ApJ, 749, 21

Akylas, A., Georgakakis, A., Georgantopoulos, I., Brightman, M., \& Nandra, K. 2012, A\&A, 546, A98

Arnaud, K. A. 1996, Astronomical Data Analysis Software and Systems V, 101, 17

Asmus, D., Gandhi, P., Hönig, S. F., Smette, A., \& Duschl, W. J. 2015, MNRAS, 454, 766

Avni, Y. 1976, ApJ, 210, 642

Ballantyne, D. R., Draper, A. R., Madsen, K. K., Rigby, J. R., \& Treister, E. 2011, ApJ, 736, 56

Baloković, M., Comastri, A., Harrison, F. A., et al. 2014, ApJ, 794, 111

Beckmann, V., Soldi, S., Ricci, C., et al. 2009, A\&A, 505, 417

Bohlin, R. C., Savage, B. D., \& Drake, J. F. 1978, ApJ, 224, 132

Boorman, P. G., Gandhi, P., Alexander, D. M., et al. 2016, ApJ, 833, 245

Burlon, D., Ajello, M., Greiner, J., et al. 2011, ApJ, 728, 58

Chiang, C.-Y., Cackett, E. M., Gandhi, P., \& Fabian, A. C. 2013, MNRAS, 430, 2943

Comastri, A. 2004, Supermassive Black Holes in the Distant Universe, 308, 245 Comastri, A., Gilli, R., Marconi, A., Risaliti, G., \& Salvati, M. 2015, A\&A, 574, L10

Della Ceca, R., Severgnini, P., Caccianiga, A., et al. 2008, Mem. Soc. Astron. It., 79,65

Del Moro, A., Alexander, D. M., Aird, J. A., et al. 2017, ApJ, 849, 57

Farrah, D., Baloković, M., Stern, D., et al. 2016, ApJ, 831, 76

Feltre, A., Hatziminaoglou, E., Fritz, J., \& Franceschini, A. 2012, MNRAS, 426, 120

Franceschini, A., Bassani, L., Cappi, M., et al. 2000, A\&A, 353, 910

Fritz, J., Franceschini, A., \& Hatziminaoglou, E. 2006, MNRAS, 366, 767

Frontera, F., Orlandini, M., Landi, R., et al. 2007, ApJ, 666, 86

Gandhi, P., Horst, H., Smette, A., et al. 2009, A\&A, 502, 457

Gandhi, P., Lansbury, G. B., Alexander, D. M., et al. 2014, ApJ, 792, 117

Gilli, R., Comastri, A., \& Hasinger, G. 2007, A\&A, 463, 79

Guainazzi, M., Matt, G., \& Perola, G. C. 2005, A\&A, 444, 119

Harrison, F. A., Craig, W. W., Christensen, F. E., et al. 2013, ApJ, 770, 103

Heckman, T. M., Ptak, A., Hornschemeier, A., \& Kauffmann, G. 2005, ApJ, 634, 161

Iwasawa, K., Fabian, A. C., \& Ettori, S. 2001, MNRAS, 321, L15

LaMassa, S. M., Heckman, T. M., Ptak, A., et al. 2011, ApJ, 729, 52

Lansbury, G. B., Gandhi, P., Alexander, D. M., et al. 2015, ApJ, 809, 115

Lansbury, G. B., Stern, D., Aird, J., et al. 2017a, ApJ, 836, 99

Lansbury, G. B., Alexander, D. M., Aird, J., et al. 2017b, ApJ, 846, 20

Lanzuisi, G., Piconcelli, E., Fiore, F., et al. 2009, A\&A, 498, 67

Lanzuisi, G., Ranalli, P., Georgantopoulos, I., et al. 2015a, A\&A, 573, A137

Lanzuisi, G., Perna, M., Delvecchio, I., et al. 2015b, A\&A, 578, A120

Lusso, E., Comastri, A., Simmons, B. D., et al. 2012, MNRAS, 425, 623

Malizia, A., Bassani, L., Bazzano, A., et al. 2012, MNRAS, 426, 1750

Marchesi, S., Ajello, M., Marcotulli, L., et al. 2018, ApJ, 854, 49

Marconi, A., Risaliti, G., Gilli, R., et al. 2004, MNRAS, 351, 169

Martocchia, S., Piconcelli, E., Zappacosta, L., et al. 2017, A\&A, 608, A51

Masini, A., Comastri, A., Baloković, M., et al. 2016, A\&A, 589, A59

Matt, G. 2000, A\&A, 355, L31

Mulchaey, J. S., Koratkar, A., Ward, M. J., et al. 1994, ApJ, 436, 586 
A\&A 619, A16 (2018)

Murphy, K. D., \& Yaqoob, T. 2009, MNRAS, 397, 1549

Oh, K., Koss, M., Markwardt, C. B., et al. 2018, ApJS, 235, 4

Panessa, F., Bassani, L., Cappi, M., et al. 2006, A\&A, 455, 173

Piconcelli, E., Fiore, F., Nicastro, F., et al. 2007, A\&A, 473, 85 (P07)

Piconcelli, E., Vignali, C., Bianchi, S., et al. 2015, A\&A, 574, L9

Ranalli, P., Comastri, A., \& Setti, G. 2003, A\&A, 399, 39

Ricci, C., Ueda, Y., Koss, M. J., et al. 2015, ApJ, 815, L13

Seaton, M. J. 1979, MNRAS, 187, 73P

Severgnini, P., Caccianiga, A., DellaCeca, R., et al. 2011, A\&A, 525, A38

Severgnini, P., Caccianiga, A., \& Della Ceca, R. 2012, A\&A, 542, A46
Stern, D. 2015, ApJ, 807, 129

Stern, D., Lansbury, G. B., Assef, R. J., et al. 2014, ApJ, 794, 102

Treister, E., Urry, C. M., \& Virani, S. 2009, ApJ, 696, 110

Tueller, J., Mushotzky, R. F., Barthelmy, S., et al. 2008, ApJ, 681, 113

Vasudevan, R. V., Mushotzky, R. F., \& Gandhi, P. 2013, ApJ, 770, L37

Veilleux, S., Kim, D.-C., Sanders, D. B., Mazzarella, J. M., \& Soifer, B. T. 1995, ApJS, 98, 171

Vignali, C. 2014, Multiwavelength AGN Surveys and Studies, 304, 132

Vignali, C., Piconcelli, E., Lanzuisi, G., et al. 2011, MNRAS, 416, 2068 (V11)

Zappacosta, L., Comastri, A., Civano, F., et al. 2018, ApJ, 854, 33 